\title{
Erratum to: Clinical utility of routine laboratory testing to identify possible secondary causes in older men with osteoporosis: the osteoporotic fractures in men (MrOS) study
}

\author{
H. A. Fink ${ }^{1}$ - S. Litwack-Harrison ${ }^{2}$ - B. C. Taylor ${ }^{3}$ - D. C. Bauer ${ }^{4}$ - E. S. Orwoll ${ }^{5}$. \\ C. G. Lee ${ }^{6} \cdot$ E. Barrett-Connor ${ }^{7}$ - J. T. Schousboe ${ }^{8}$ - D. M. Kado ${ }^{7}$ P. S. Garimella ${ }^{9}$ • \\ K. E. Ensrud ${ }^{10}$ - For the Osteoporotic Fractures in Men (MrOS) Study Group
}

Published online: 20 October 2016

(C) International Osteoporosis Foundation and National Osteoporosis Foundation 2016

\section{Erratum to: Osteoporos Int \\ DOI 10.1007/s00198-015-3356-y}

Owing to an oversight by the authors, the 24-h urine calcium results reported as milligrams urinary calcium per $24 \mathrm{~h}$ in this article were actually milligrams urinary calcium per deciliter:

As a result, the statement in the final sentence in the "Results" section of the abstract that "No osteoporotic men had hypercalciuria" was incorrect. The last two sentences of this paragraph should have read "Hypercortisolism, hyperthyroidism, and hypercalciuria were uncommon and not significantly more frequent in men with osteoporosis." Furthermore, the statement in the "Results" section of the main text that "The only man with urine calcium $>300 \mathrm{mg} / 24 \mathrm{~h}$ did not have osteoporosis" was incorrect. The paragraph containing this sentence should have read "In the smaller sample of men with available 24-h urine measures, neither hypercortisolism defined as UFC $>100 \mathrm{mcg} / 24 \mathrm{~h}$ nor hypercalciuria defined as urine calcium $>300 \mathrm{mg} / 24 \mathrm{~h}$ was significantly more frequent in men with than in those without osteoporosis (Table 2)." Finally, the results reported for "Hypercalciuria, $>300 \mathrm{mg}$ calcium $/ 24 \mathrm{~h}$ " under the heading "Primary definitions of laboratory abnormalities" in Table 2 were incorrect; the corrected Table 2 is shown here in full.

The authors apologize for any inconvenience caused.

The online version of the original article can be found at http://dx.doi. org/10.1007/s00198-015-3356-y

H. A. Fink

Howard.fink@va.gov

1 Geriatric Research Education \& Clinical Center, Minneapolis VA, Health Care System, One Veterans Drive, 11-G, Minneapolis, MN 55417, USA

2 Department of Epidemiology \& Statistics, University of California, San Francisco, San Francisco Coordinating Center, Mission Hall: Global Health \& Clinical Sciences Building, 550 16th Street, 2nd, floor, Box, San Francisco, CA \#0560, USA

3 Center for Chronic Disease Outcomes Research, Minneapolis VA Health Care System, One Veterans Drive, Mail code 152, Building 9, Minneapolis, MN 55417, USA

4 Department of Medicine, University of California, San Francisco, 1545 Divisadero St, 3rd Floor, San Francisco, CA, USA
5 Bone \& Mineral Unit, Department of Medicine, Oregon Health \& Science University, 3181 SW Sam Jackson Park Road, CR113, Portland, OR 97239, USA

6 Portland Veterans Affairs HealthCareSystem, 3710SWUSVeterans Hospital Rd, R\&D45, Portland, OR 97239, USA

7 Department of Family Medicine \& Public Health, University of California, San Diego 9500 Gilman Drive, La Jolla, CA 92093, USA

8 Health Research Center, Park Nicollet Institute for Research and Education, 3800 Park Nicollet Boulevard, Minneapolis, MN 55416, USA

9 Division of Nephrology, Tufts Medical Center, 800 Washington, Street, Box 391, Boston, MA 02111, USA

10 Division of General Internal Medicine, Minneapolis VA Health Care System, One Veterans Drive, 111-0, Minneapolis, MN 55417, USA 
Table 2 Relative prevalence of selected laboratory abnormalities in men with and without osteoporosis, PR (95\% CI)

\begin{tabular}{|c|c|c|c|c|}
\hline Diagnosis & $\begin{array}{l}\text { Osteoporosis, } \\
\%(n)\end{array}$ & $\begin{array}{l}\text { No osteoporosis, } \\
\%(n)\end{array}$ & $\begin{array}{l}\text { PR } \\
(95 \% \mathrm{CI})\end{array}$ & $\begin{array}{l}\text { NNTB } \\
(95 \% \mathrm{CI})\end{array}$ \\
\hline \multicolumn{5}{|l|}{ Primary definitions of laboratory abnormalities } \\
\hline \multicolumn{5}{|l|}{$\begin{array}{l}\text { Cohort with random baseline serum and } \\
\text { spot urine }(n=1572)\end{array}$} \\
\hline $25(\mathrm{OH})$ vitamin D deficiency $<20 \mathrm{ng} / \mathrm{mL}$ & $30.7(50)$ & $24.7(348)$ & $1.24(0.97-1.59)$ & 16.7 (NNTB 7.5 to $\infty$ to NNTH 68.0 ) \\
\hline $\begin{array}{l}\text { Kidney disease, eGFRcr }<60 \mathrm{~mL} / \mathrm{min} / 1.73 \mathrm{~m}^{2} \\
\text { or urine ACR } \geq 30 \mathrm{mg} / \mathrm{g}\end{array}$ & $17.1(25)$ & $17.9(239)$ & $0.96(0.66-1.39)$ & $-125.0($ NNTB 17.6 to $\infty$ to NNTH 13.8) \\
\hline Hyperthyroidism, TSH $<0.55 \mathrm{mIU} / \mathrm{L}$ & $2.5(4)$ & $2.8(39)$ & $0.89(0.32-2.46)$ & -333.3 (NNTB 44.7 to $\infty$ to NNTH 35.2 ) \\
\hline Low testosterone, $<200 \mathrm{ng} / \mathrm{dL}$ & $7.6(12)$ & $6.2(87)$ & $1.22(0.68-2.18)$ & 71.4 (NNTB 17.6 to $\infty$ to NNTH 33.8 ) \\
\hline Hyperparathyroidism, $\mathrm{PTH}>66 \mathrm{pg} / \mathrm{mL}$ & $6.2(10)$ & $3.4(48)$ & $1.81(0.93-3.50)$ & 35.7 (NNTB 15.2 to $\infty$ to NNTH 93.7) \\
\hline Hypercalcemia, $\mathrm{Ca}>10.4 \mathrm{mg} / \mathrm{dL}$ & $1.4(2)$ & $0.6(8)$ & $2.29(0.49-10.7)$ & $125.0($ NNTB 37.0 to $\infty$ to NNTH 86.2) \\
\hline Hyperphosphatemia, PO4 >4.7 mg/dL & $0(0)$ & $0.1(1)$ & $\mathrm{NC}$ & $\mathrm{NC}$ \\
\hline High alkaline phosphatase $>129 \mathrm{IU} / \mathrm{L}$ & $6.9(10)$ & $2.3(30)$ & $3.05(1.52-6.11)$ & $21.7(11.4$ to 233.4$)$ \\
\hline $\begin{array}{l}\text { Hypercalciuria, spot urine calcium/creatinine } \\
\text { ratio } \geq 0.3\end{array}$ & $1.2(2)$ & $0.8(11)$ & $1.57(0.35-7.01)$ & $250.0($ NNTB 45.3 to $\infty$ to NNTH 76.0 ) \\
\hline At least one of above laboratory abnormalities ${ }^{\mathrm{a}}$ & $58.3(81)$ & $56.5(740)$ & $1.03(0.89-1.20)$ & 55.6 (NNTB 9.6 to $\infty$ to NNTH 14.7 ) \\
\hline \multicolumn{5}{|l|}{ Cohort with 24 -h urine $(n=337)$} \\
\hline Hypercortisolism, UFC >100 mg/24 h & $2.9(1)$ & $1.3(4)$ & $2.16(0.25-18.8)$ & 66.7 (NNTB 13.9 to $\infty$ to NNTH 24.2) \\
\hline Hypercalciuria, $>300 \mathrm{mg}$ calcium $/ 24 \mathrm{~h}$ & $3.2(1)$ & $5.8(17)$ & $0.55(0.08-4.02)$ & -38.5 (NNTB 23.9 to $\infty$ to NNTH 10.7) \\
\hline \multicolumn{5}{|l|}{ Alternative definitions of laboratory abnormalities } \\
\hline \multicolumn{5}{|l|}{$\begin{array}{l}\text { Cohort with random baseline serum and } \\
\text { spot urine }(n=1572)\end{array}$} \\
\hline $25(\mathrm{OH})$ vitamin $\mathrm{D}$ insufficiency $<30 \mathrm{ng} / \mathrm{mL}$ & $84.1(137)$ & $74.1(1043)$ & $1.13(1.05-1.22)$ & $10.0(6.2-25.6)$ \\
\hline Low testosterone $<300 \mathrm{ng} / \mathrm{dL}$ & $25.3(40)$ & $26.3(367)$ & $0.96(0.73-1.28)$ & $-100.0($ NNTB 16.2 to $\infty$ to NNTH 12.3$)$ \\
\hline $\begin{array}{l}\text { Hypercalciuria, spot urine calcium/creatinine } \\
\text { ratio } \geq 0.2\end{array}$ & $5.6(9)$ & $3.6(50)$ & $1.55(0.78-3.10)$ & $50.0(\mathrm{NNTB} 17.7$ to $\infty$ to NNTH 59.4$)$ \\
\hline \multicolumn{5}{|l|}{ Cohort with 24-h urine $(n=337)$} \\
\hline Hypercortisolism, UFC $>50 \mathrm{mg} / 24 \mathrm{~h}$ & $5.7(2)$ & $7.3(22)$ & $0.78(0.19-3.20)$ & -62.5 (NNTB 15.0 to $\infty$ to NNTH 10.2) \\
\hline
\end{tabular}

There were 122 participants with missing laboratory data for at least one measure, including the following number with missing data for specific laboratory tests: eGFRcr 91, serum calcium 91, serum phosphorus 91, serum alkaline phosphatase 91, testosterone 18, spot urine calcium 13, PTH 4, TSH 2, 25(OH) vitamin D 1, 24-h UFC 9, and 24-h urine calcium 21

$P R$ prevalence ratio, NNTB number needed to test (benefit), NNTH number needed to treat (harm), $P T H$ parathyroid hormone, TSH thyroid-stimulating hormone, eGFRcr estimated creatinine-derived glomerular filtration rate, ACR urinary albumin/creatinine ratio, UFC urine free cortisol, $N C$ not calculable

${ }^{a}$ Defined by presence of any of the following: $25(\mathrm{OH})$ vitamin $\mathrm{D}<20 \mathrm{ng} / \mathrm{mL}$, kidney disease (eGFRcr $<60$ or ACR $\geq 30$ ), TSH $<0.55 \mathrm{mIU} / \mathrm{L}$, total testosterone $<200 \mathrm{ng} / \mathrm{dL}, \mathrm{PTH}>66 \mathrm{pg} / \mathrm{mL}$, serum calcium $>10.4 \mathrm{mg} / \mathrm{dL}$, serum phosphorus $>4.7 \mathrm{mg} / \mathrm{dL}$, serum alkaline phosphatase $>129 \mathrm{IU} / \mathrm{L}$, or spot urine calcium/creatinine ratio $\geq 0.3$ 